\title{
Outcomes with Cefiderocol Treatment in Patients with Bacteraemia Enrolled into Prospective Phase 2 and Phase 3 Randomised Clinical Studies
}

\author{
David L. Paterson · Masahiro Kinoshita · Takamichi Baba • \\ Roger Echols · Simon Portsmouth
}

Received: December 8, 2021 / Accepted: January 26, 2022 / Published online: February 20, 2022

(C) The Author(s) 2022

\section{ABSTRACT}

Introduction: A post hoc, descriptive analysis of three prospective, randomised, controlled clinical studies investigating cefiderocol in gram-negative bacterial infections was conducted to assess its efficacy in patients with baseline bacteraemia.

Methods: Data from APEKS-cUTI (NCT02321800), APEKS-NP (NCT03032380) and CREDIBLE-CR (NCT02714595) studies were assessed individually. Patients received cefiderocol $2 \mathrm{~g}$, q8h, for 7-14 days or comparators (imipenem/cilastatin [APEKS-cUTI], meropenem [APEKS-NP] or best available therapy [BAT; CREDIBLE-CR]). Bacteraemia and clinical

Supplementary Information The online version contains supplementary material available at https:// doi.org/10.1007/s40121-022-00598-9.

\section{L. Paterson}

Centre for Clinical Research, Faculty of Medicine, The University of Queensland, Royal Brisbane and Women's Hospital Campus, Brisbane, Australia

M. Kinoshita · T. Baba

Shionogi \& Co., Ltd., Osaka, Japan

R. Echols

Infectious Disease Drug Development Consulting, LLC, Easton, CT, USA

S. Portsmouth $(\varangle)$

Shionogi Inc., 300 Campus Park, Florham Park, NJ 07932, USA

e-mail: Simon.Portsmouth@shionogi.com outcomes were assessed at early assessment (EA), end of treatment (EOT) and test of cure (TOC) for patients in the intention-to-treat populations with baseline blood samples positive for aerobic gram-negative species. Eradication, persistence or recurrence of baseline blood pathogen was confirmed from follow-up blood cultures; in the absence of follow-up blood cultures, clinical response, administration of additional antibiotics and vital status were used to assess bacteraemia outcome.

Results: Of 885 patients randomised, 84 had bacteraemia and 89 (cefiderocol: 55, comparators: 34) gram-negative pathogens were isolated, namely Enterobacterales $(n=62)$ and non-fermenters $(n=27)$. At EA, on-therapy bacteraemia eradication rates in APEKS-cUTI, APEKS-NP and CREDIBLE-CR were 100\% (19/ 19), $50.0 \%(4 / 8)$ and $72.0 \%(18 / 25)$ with cefiderocol. Corresponding rates for comparators were $77.8 \%(7 / 9), 100 \%(10 / 10)$ and $69.2 \%$ (9/13), respectively. Persistence in blood at EA was seen in six patients overall (cefiderocol: 3, comparators: 3); indeterminate responses were common (cefiderocol: 8, comparators: 3), usually due to lack of blood cultures. Clinical cure/ improvement rates at EA in APEKS-cUTI, APEKS$\mathrm{NP}$ and CREDIBLE-CR were 100\% (19/19), $62.5 \%(5 / 8)$ and $64.0 \%(16 / 25)$ with cefiderocol. Corresponding rates for comparators were $77.8 \%$ (7/9), 90.0\% (9/10) and 30.8\% (4/13), respectively. Bacteraemia eradication rates with cefiderocol in APEKS-cUTI, APEKS-NP and 
CREDIBLE-CR were $89.5 \%, 37.5 \%$ and $60.0 \%$ at EOT and $78.9 \%, 12.5 \%$ and $44.0 \%$ at TOC.

Conclusion: This descriptive analysis suggests that cefiderocol may be a useful treatment option for gram-negative bacteraemia, including pathogens resistant to other antibiotics.

Keywords: Bloodstream infection; Carbapenem resistance; Cefiderocol; Eradication; ESBL; Gram-negative bacteraemia; Metallo-beta-lactamase; Multidrug resistance

\section{Key Summary Points}

\section{Why carry out this study?}

Randomised clinical trials in infectious diseases routinely obtain blood cultures at baseline; however, follow-up blood cultures to determine bacterial clearance from blood at later time points are often not systematically evaluated.

The objective of this post hoc analysis of three randomised clinical trials was to investigate clearance of gram-negative bacteria from blood in patients who were treated with cefiderocol (and comparator antibiotics).

\section{What did the study ask?}

This post hoc descriptive analysis used data from three prospective randomised clinical trials involving patients with complicated urinary tract infections (cUTI), nosocomial pneumonia (NP) and serious carbapenem-resistant (CR) infections to assess the clinical and microbiological efficacy of the siderophore cephalosporin cefiderocol (and comparators) in patients with baseline bacteraemia.

\section{What were the outcomes/conclusions?}

Cefiderocol rapidly cleared bacteraemia due to aerobic gram-negative pathogens, including both Enterobacterales and nonfermenters, in most patients, including isolates that were often CR and/or expressed an extended-spectrum betalactamase (ESBL) enzyme; persistence and recurrence occurred infrequently and were related to inadequate source control.

\section{What was learned from the study?}

The species of pathogens causing bacteraemia were closely related to the primary infection type, that is: Escherichia coli (cUTI), Klebsiella pneumoniae (NP) and Acinetobacter spp. (bloodstream infection/ sepsis).

This analysis suggests that cefiderocol may be a treatment option for gram-negative bacteraemia.

Further randomised clinical trials to define the treatment benefit of cefiderocol for gram-negative bacteraemia are indicated.

\section{INTRODUCTION}

Bloodstream infections (BSIs) are an important cause of morbidity and mortality across all ages [1-4]. BSI is a relatively common complication of serious infections [5-7], and outcomes in patients with gram-negative bacteraemia are often poor [8], particularly when initiation of appropriate antimicrobial therapy is delayed [9-11]. Equally important to effective management is adequate infection source control $[9,12]$.

Frequent clinically relevant pathogens include Enterobacterales, particularly Escherichia coli and Klebsiella pneumoniae [1, 6], and the non-fermenters Pseudomonas aeruginosa, Acinetobacter baumannii and Stenotrophomonas maltophilia [13-15]. With rising global antimicrobial resistance, the management of BSIs 
caused by carbapenem-resistant (CR), multidrug-resistant (MDR) or extensively drug-resistant (XDR) Enterobacterales and nonfermenters [8, 9, 14-19] has become challenging in both community and hospital settings $[14,15]$. MDR gram-negative pathogens are also a common cause of neonatal sepsis in lowerand middle-income countries, contributing to infant morbidity and mortality $[20,21]$. Antibiotic resistance is the result of a range of mechanisms employed by various species, including plasmid-encoded extended-spectrum beta-lactamases (ESBLs, e.g. Temoneira extended-spectrum beta-lactamase [TEM]-, sulphydryl variant of TEM extended-spectrum beta-lactamase [SHV]- and cefotaxime-hydrolysing beta-lactamase [CTX-M]-type enzymes), carbapenemases (e.g. K. pneumoniae carbapenemase [KPC]), metallo-beta-lactamases (MBLs, e.g. imipenemase metallo-beta-lactamase [IMP], Verona integron-encoded metallobeta-lactamase [VIM], New Delhi metallo-betalactamase $[\mathrm{NDM}]$ ) and oxacillinase (OXA) enzymes [19]. Due to the variability of resistance mechanisms, appropriate antibiotic treatment is often delayed, increasing the risk of mortality [9-11].

Prospective randomised clinical trials of patients with gram-negative BSI are uncommon. Data from subset analyses of patients with bacteraemia from infection site-specific trials are alternative evidence, but often limited because of the general exclusion of patients with highly resistant pathogens. Additionally, because these studies were generally focused on specific infection sites (e.g. lung, urinary tract), assessment of the microbiological clearance of the baseline blood pathogen is not systematically conducted. Nevertheless, since bacteraemia represents the most severe manifestation of infection, even if the primary source is identified, failure to clear culturable bacteraemia is considered a suboptimal response to treatment. Among patients with gram-negative bacteraemia, positive follow-up blood cultures may be associated with increased mortality compared with negative blood cultures [22]. Thus, there is a need to more precisely evaluate the treatment of secondary bacteraemia caused by resistant gram-negative pathogens.
Cefiderocol is a siderophore cephalosporin antibiotic, with activity against a wide range of aerobic gram-negative bacteria, including those associated with bacteraemia, such as CR, ESBLproducing or MDR strains of Enterobacterales, $P$. aeruginosa, A. baumannii and S. maltophilia [23-25]. The efficacy and safety of cefiderocol have been demonstrated in prospective randomised Phase 2 and Phase 3 clinical studies of hospitalised patients with complicated urinary tract infection (cUTI; APEKS-cUTI) [26], hospital-acquired and ventilator-associated pneumonia (APEKS-NP) [27] and infections caused by CR organisms (the pathogen-focused CREDIBLE-CR study) [28].

We conducted a post hoc descriptive analysis of data from APEKS-cUTI, APEKS-NP and CREDIBLE-CR, which include patients with documented secondary gram-negative bacteraemia, to describe clinical and microbiological bacteraemia outcomes following intravenous cefiderocol treatment for 7-14 days in patients with gram-negative bacteraemia. We have incorporated an algorithm to assess bacteraemia outcomes based on the presence or absence of follow-up blood samples, clinical outcome, vital status and administration of additional antibiotics.

\section{METHODS}

\section{Study Designs and Participants}

The current analysis included data from three prospective, multicentre, randomised trials, the study designs and primary results of which have been described in detail elsewhere [26-28]. Briefly, APEKS-cUTI (NCT02321800) was a 2:1 randomised, double-blind, non-inferiority Phase 2 study conducted in 67 hospitals across 15 countries in patients with cUTI or acute uncomplicated pyelonephritis and compared cefiderocol ( $2 \mathrm{~g}$, q8h, 1-h infusion) with imipenem-cilastatin $(1 \mathrm{~g} / 1 \mathrm{~g}$, three-times daily, 1-h infusion). Patients with known carbapenemnon-susceptible pathogens were excluded. No adjunctive gram-negative therapy was allowed [26]. APEKS-NP (NCT03032380) was a 1:1 randomised, double-blind, non-inferiority Phase 3 
study in patients with nosocomial pneumonia (NP), comparing cefiderocol $(2 \mathrm{~g}$, q8h, 3-h infusion) with high-dose, extended-infusion meropenem ( $2 \mathrm{~g}, \mathrm{q} 8 \mathrm{~h}, 3 \mathrm{~h}$ infusion). No adjunctive gram-negative therapy was allowed; however, linezolid $600 \mathrm{mg}$, twice daily, was administered for at least 5 days in both arms to cover gram-positive bacteria in the cefiderocol arm and methicillin-resistant Staphylococcus aureus in both arms. Patients with CR pathogens known at the time of randomisation were excluded [27]. CREDIBLE-CR (NCT02714595) was a 2:1 randomised, open-label, descriptive Phase 3 study conducted in patients with serious infection (cUTI, NP and BSI/sepsis) caused by CR gram-negative pathogens. Patients were treated with cefiderocol ( $2 \mathrm{~g}$, q8h, 3-h infusion) or best available therapy (BAT, which could include up to three agents dosed according to the local label) [28]. In all three studies, eradication of pathogen-causing bacteraemia was confirmed from subsequent blood cultures collected at early assessment (EA), end of treatment (EOT), test of cure (TOC) and follow-up (FUP) visits.

Blood cultures at randomisation (baseline) and post-randomisation were collected in each study for identification and susceptibility testing by the hospital laboratory. In all three studies, two blood cultures from separate venepunctures were collected within $48 \mathrm{~h}$ prior to start of study drug treatment at randomisation, and if a blood culture was positive for a gram-negative pathogen, subsequent blood samples were to be collected at EA, EOT, TOC and/or FUP visits or until negative. Species considered to be pathogens were sent to a central reference laboratory for confirmation of species identification, susceptibility testing and molecular characterisation of resistance genes [26-28].

Bacterial isolates identified by the local laboratory from blood cultures were analysed by the central laboratory (JMI Labs, North Liberty, IA, USA [APEKS-cUTI] [26], IHMA Schaumburg, IL, USA [APEKS-NP and CREDIBLE-CR] [27, 28]) for species identification and determination of minimum inhibitory concentrations (MICs) to study drugs and other antibiotics. In addition, the ESBL status of original pathogens from urine were confirmed in isolates in APEKS-cUTI [26], and molecular mechanisms for isolates with cefepime or aztreonam resistance were determined for respiratory isolates in APEKS-NP [27] and for all isolates in CREDIBLE-CR [28].

\section{Key Outcomes}

The key outcomes of this descriptive, post hoc analysis were bacteraemia outcome and clinical outcome per patient at EA, EOT and TOC. Other endpoints were bacteraemia outcome and clinical outcome by pathogen and all-cause mortality (ACM) at Day 14 and Day 28. Outcome according to resistance mechanism was also investigated. Definitions for primary clinical and microbiological outcomes in each of the studies are provided elsewhere [26-28].

The clinical outcome in this analysis was determined using the primary data set in each of the three studies [26-28]. In brief, clinical cure was defined as improvement of clinical signs and symptoms without the need for additional antibiotics. Clinical failure was defined as persistent signs and symptoms requiring additional antibiotics or death due to the primary infection. Indeterminate clinical response was defined as clinical outcome unable to be determined because of loss to follow-up or due to death unrelated to the primary infection.

Bacteraemia microbiological outcome comprised eradication, presumed eradication, persistence, recurrence and indeterminate responses at each time point (Table 1). Where available, post-randomisation blood cultures were used to determine eradication, persistence or recurrence outcome. Where there was no post-randomisation blood culture, clinical response, use of additional antibiotic and vital status were used to assess microbiological bacteraemia outcome. Bacteraemia outcome was an indeterminate response if patients had received additional antibiotics regardless of their clinical outcome at the same study visit or had missing blood cultures with clinical failure or clinical indeterminate response or had died (Table 1). 
Table 1 Post hoc analysis determination of bacteraemia outcome

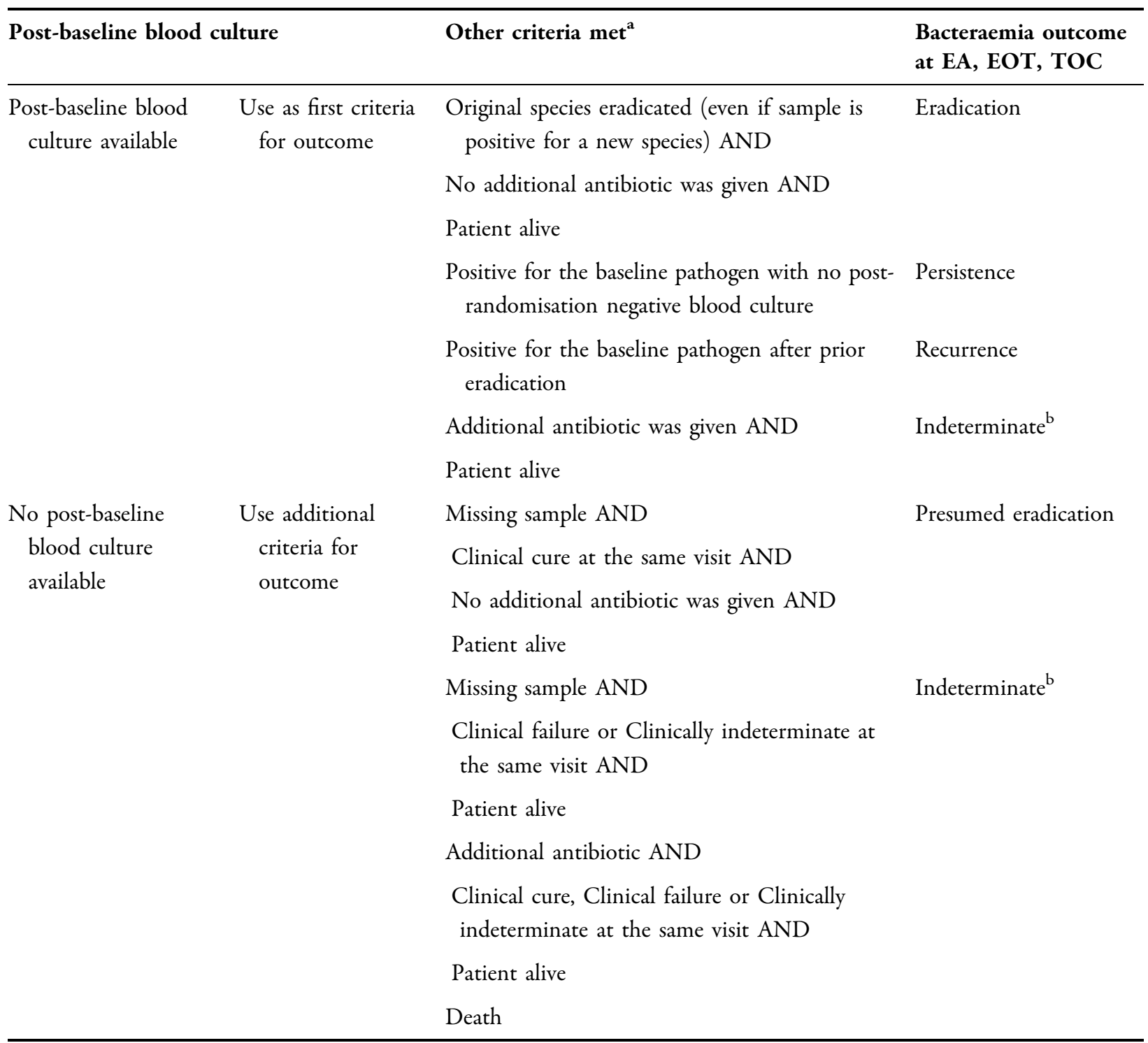

$E A$ early assessment, EOT end of treatment, TOC test of cure

${ }^{\mathrm{a}}$ For each time point, the outcome is defined by the criteria met at that time point

${ }^{\mathrm{b}}$ Indeterminate bacteraemia response was not carried forward for patients alive

\section{Ethics}

Data from all three studies included in this post hoc analysis have been reported elsewhere [26-28]. For each trial, the study protocol was approved by the institutional review board or ethics committee at each participating centre and complied with International Conference on Harmonisation Guideline for Good Clinical
Practice and the Helsinki Declaration of 1964 and its later amendments. All patients or their representatives provided written informed consent according to local guidelines.

\section{Statistics}

In this post hoc analysis, only descriptive summary statistics for individual studies are 
presented. Data are not integrated across studies because of the differences in study designs, enrolment criteria and patient populations. Data were analysed using SAS version 9.2 or higher.

Data collections were previously pre-specified for each study for demographics and baseline clinical characteristics, and missing data were not collected retrospectively. Although the main focus of the analysis was to describe the clinical effectiveness and microbiological activity of cefiderocol in patients with gram-negative bacteraemia, effects of comparator antibiotics from each of the three studies involved were also included for completeness. However, no statistical comparisons between cefiderocol and comparator antibiotics were made for this analysis. Only descriptive summary statistics are provided for all agents. Categorical values (sex, race, region, creatinine clearance category, infection site, bacteraemia outcome, clinical outcome, ESBL status, resistance mechanism, vital status, Acute Physiology and Chronic Health Evaluation II [APACHE II] score, Sequential Organ Failure Assessment [SOFA] score) are summarised with the number of patients or pathogens within each category and percentage, and continuous values (age, creatinine clearance, APACHE II score, SOFA score) are described with mean (standard deviation $[\mathrm{SD}]$ ) and median (range [minimum, maximum]).

The analysed population per study is the intention-to-treat (ITT) population, in which patients in both treatment arms received at least one dose of study drug. Patients with NP or cUTI diagnosis could have had no primary infection gram-negative pathogen but a positive blood culture at randomisation. ACM was calculated for the ITT population in each study.

\section{RESULTS}

\section{Patient Disposition}

Across the ITT populations of the studies, 84 of 885 randomised patients $(9.5 \%)$ had positive baseline blood cultures at randomisation (cefiderocol 52, comparators 32), with 89 gram- negative pathogens isolated (cefiderocol 55, comparators 34) (Fig. 1). Two patients included in this analysis, both from CREDIBLE-CR (one in each treatment arm), also had gram-positive pathogens in the blood (Fig. 1).

Most patients were enrolled in Europe (Table 2). The primary infections across 84 patients were cUTI $(n=31$ [36.9\%]), respiratory $(n=22[26.2 \%])$ and BSI/sepsis $(n=31$ [36.9\%]). Bacteraemia secondary to cUTI or NP was observed in $19 / 290$ patients $(6.6 \%)$ in the cefiderocol arm and 9/147 patients (6.1\%) in the comparator arm in APEKS-cUTI, and in 8/148 (5.4\%) and 10/150 (6.7\%) patients, respectively, in APEKS-NP. In CREDIBLE-CR, $24.8 \%$ of patients in the cefiderocol arm and $26.5 \%$ in the BAT arm had bacteraemia at randomisation, including patients primarily enrolled with BSI/sepsis diagnosis, and three patients with cUTI and four patients with NP diagnosed at randomisation (Table 2).

Among Enterobacterales $(n=62), \quad$ E. coli $(n=29)$ and K. pneumoniae $(n=23)$ were most frequently identified in blood cultures, while among non-fermenters $(n=27)$, the most frequent species were Acinetobacter spp. $(n=21)$ (Table 3). The most frequent pathogen was E. coli $(85.7 \%$ [24/28 patients]) in APEKS-cUTI and $K$. pneumoniae $(38.9 \%$ [7/18 patients]) in APEKS-NP, and non-fermenters causing bacteraemia were infrequent in these two studies. In CREDIBLE-CR, Acinetobacter spp. and K. pneumoniae were the two most frequently isolated pathogens (Table 3). Among patients with cUTI primary diagnosis in CREDIBLE-CR, two patients had K. pneumoniae and one patient had $E$. coli in the cefiderocol arm.

Of 37 CR blood isolates at baseline, 34 were found in CREDIBLE-CR. Nineteen Enterobacterales were $\mathrm{CR}$ at baseline: 13 in patients receiving cefiderocol (CREDIBLE-CR [ $n=12$ ], APEKS-NP $[n=1])$ and 6 in patients receiving BAT (CREDIBLE-CR). Eighteen Acinetobacter spp. were CR at baseline: 11 in patients receiving cefiderocol (CREDIBLE-CR $[n=10]$, APEKS-NP $[n=1])$ and 7 in patients receiving comparators (CREDIBLE-CR $[n=6]$, APEKS-cUTI $[n=1]$ ). 

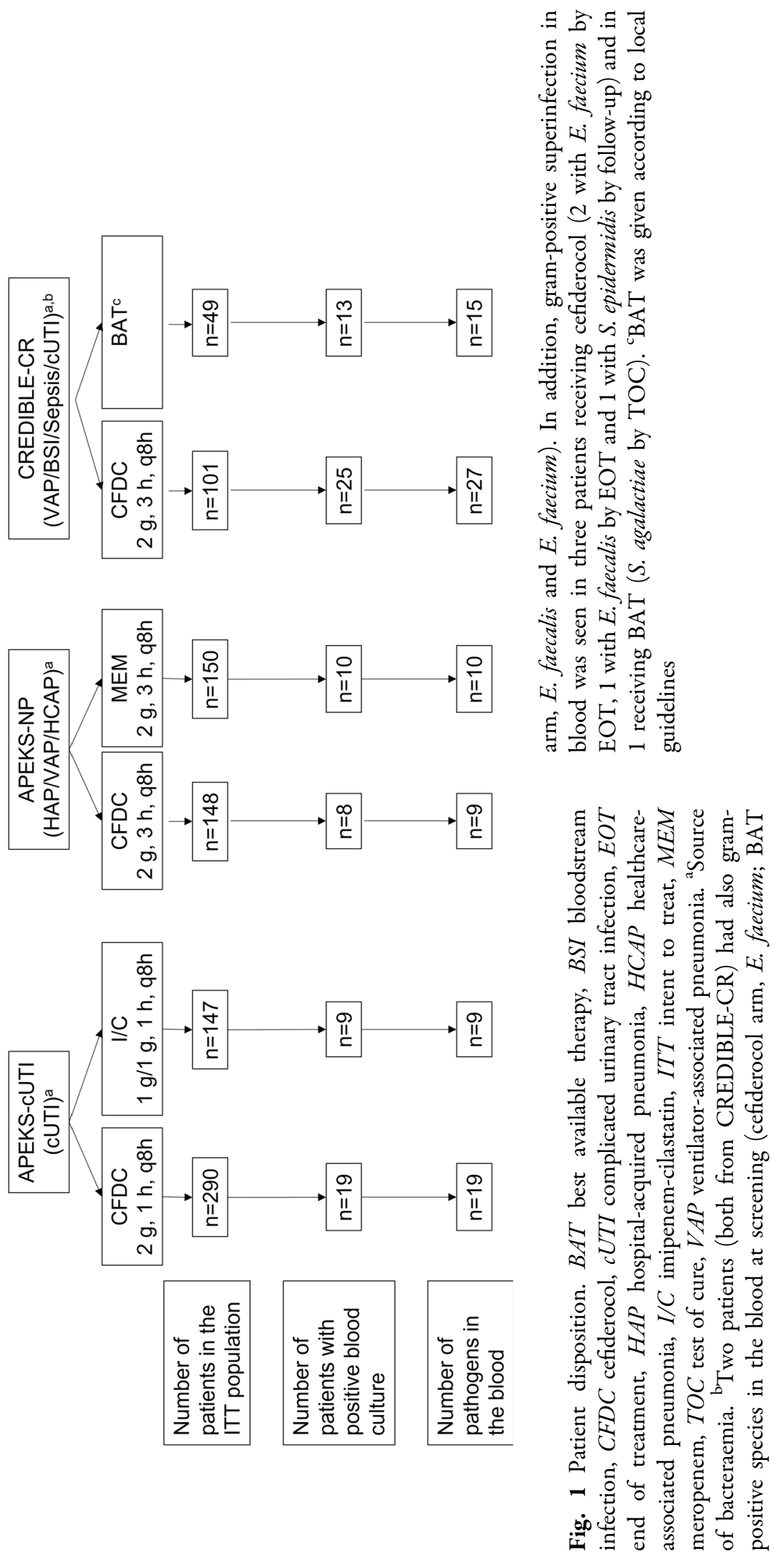
Table 2 Demographics and characteristics at baseline of patients with positive blood culture in the three studies (intentionto-treat population)

\begin{tabular}{|c|c|c|c|c|c|c|}
\hline & \multicolumn{2}{|l|}{ APEKS-cUTI } & \multicolumn{2}{|l|}{ APEKS-NP } & \multicolumn{2}{|c|}{ CREDIBLE-CR } \\
\hline & $\begin{array}{l}\text { Cefiderocol } \\
(N=19)\end{array}$ & $\begin{array}{l}\text { Imipenem/ } \\
\text { cilastatin } \\
(N=9)\end{array}$ & $\begin{array}{l}\text { Cefiderocol } \\
(N=8)\end{array}$ & $\begin{array}{l}\text { Meropenem } \\
(N=10)\end{array}$ & $\begin{array}{l}\text { Cefiderocol } \\
(N=25)\end{array}$ & $\begin{array}{l}\text { BAT } \\
(N=13)\end{array}$ \\
\hline \multicolumn{7}{|l|}{ Age } \\
\hline Mean (SD), years & $60.5(16.6)$ & $60.8(24.3)$ & $73.5(11.5)$ & $66.8(12.4)$ & $61.4(20.3)$ & $61.9(16.8)$ \\
\hline Median (range), years & $64.0(32-83)$ & $68.0(21-89)$ & $74.0(58-89)$ & $66.0(46-87)$ & $68.0(21-85)$ & $64.0(28-84)$ \\
\hline$\geq 65$ years, $n(\%)$ & $9(47.4)$ & $5(55.6)$ & $5(62.5)$ & $5(50.0)$ & $15(60.0)$ & $6(46.2)$ \\
\hline \multicolumn{7}{|l|}{ Sex, $n(\%)$} \\
\hline Male & $8(42.1)$ & $5(55.6)$ & $4(50.0)$ & $4(40.0)$ & $18(72.0)$ & $8(61.5)$ \\
\hline \multicolumn{7}{|l|}{ Region, $n$ (\%) } \\
\hline North America & 0 & 0 & $1(12.5)$ & 0 & 0 & 0 \\
\hline South America & 0 & 0 & 0 & 0 & $1(4.0)$ & 0 \\
\hline Europe & $15(78.9)$ & $7(77.8)$ & $6(75.0)$ & $9(90.0)$ & $22(88.0)$ & $12(92.3)$ \\
\hline Asia/Pacific & $4(21.1)$ & $2(22.2)$ & $1(12.5)$ & $1(10.0)$ & $2(8.0)$ & $1(7.7)$ \\
\hline \multicolumn{7}{|l|}{$\mathrm{CrCL}, \mathrm{ml} / \mathrm{min}$} \\
\hline Mean (SD) & $72.9(30.5)$ & $57.1(23.9)$ & $68.7(50.5)$ & $58.1(53.0)$ & $94.0(113.0)$ & $89.6(53.4)$ \\
\hline Median (range) & $74.0(32-130)$ & $53.0(26-112)$ & $43.9(18-139)$ & $37.1(13-197)$ & $49.6(10-540)$ & $63.2(37-216)$ \\
\hline$\geq 120, n(\%)$ & $3(15.8)$ & 0 & $2(25.0)$ & $1(10.0)$ & $5(20.0)$ & $3(23.1)$ \\
\hline$>80-<120, n(\%)$ & $3(15.8)$ & $1(11.1)$ & $1(12.5)$ & $1(10.0)$ & $4(16.0)$ & $2(15.4)$ \\
\hline$>50-80, n(\%)$ & $8(42.1)$ & $5(55.6)$ & $1(12.5)$ & $2(20.0)$ & $3(12.0)$ & $5(38.5)$ \\
\hline $30-50, n(\%)$ & $5(26.3)$ & $2(22.2)$ & $2(25.0)$ & $3(30.0)$ & $7(28.0)$ & $3(23.1)$ \\
\hline$<30, n(\%)$ & 0 & $1(11.1)$ & $2(25.0)$ & $3(30.0)$ & $6(24.0)$ & 0 \\
\hline \multicolumn{7}{|l|}{ APACHE II } \\
\hline Mean (SD) & $\mathrm{n} / \mathrm{a}$ & $\mathrm{n} / \mathrm{a}$ & $20.5(9.1)$ & $20.3(8.5)$ & $16.4(6.0)$ & $17.1(6.3)$ \\
\hline Median (range) & $\mathrm{n} / \mathrm{a}$ & $\mathrm{n} / \mathrm{a}$ & $19.5(10-34)$ & $20.5(8-35)$ & $15.0(5-29)$ & $18.0(6-26)$ \\
\hline$\geq 16, n(\%)$ & $\mathrm{n} / \mathrm{a}$ & $\mathrm{n} / \mathrm{a}$ & $5(62.5)$ & $7(70.0)$ & $12(48.0)$ & $7(53.8)$ \\
\hline \multicolumn{7}{|l|}{ SOFA } \\
\hline Mean (SD) & & & & & & \\
\hline
\end{tabular}


Table 2 continued

\begin{tabular}{|c|c|c|c|c|c|c|}
\hline & \multicolumn{2}{|c|}{ APEKS-cUTI } & \multicolumn{2}{|l|}{ APEKS-NP } & \multicolumn{2}{|c|}{ CREDIBLE-CR } \\
\hline & $\begin{array}{l}\text { Cefiderocol } \\
(N=19)\end{array}$ & $\begin{array}{l}\text { Imipenem/ } \\
\text { cilastatin } \\
(N=9)\end{array}$ & $\begin{array}{l}\text { Cefiderocol } \\
(N=8)\end{array}$ & $\begin{array}{l}\text { Meropenem } \\
(N=10)\end{array}$ & $\begin{array}{l}\text { Cefiderocol } \\
(N=25)\end{array}$ & $\begin{array}{l}\text { BAT } \\
(N=13)\end{array}$ \\
\hline Ventilated & $\mathrm{n} / \mathrm{a}$ & $\mathrm{n} / \mathrm{a}$ & $9.3(4.1)$ & $8.4(2.6)$ & $6.5(4.1)$ & $6.5(4.8)$ \\
\hline Non-ventilated & $\mathrm{n} / \mathrm{a}$ & $\mathrm{n} / \mathrm{a}$ & $3.0(1.8)$ & $4.7(3.1)$ & & \\
\hline \multicolumn{7}{|l|}{ Median (range) } \\
\hline Ventilated & $\mathrm{n} / \mathrm{a}$ & $\mathrm{n} / \mathrm{a}$ & $10.0(4-13)$ & $9.0(4-11)$ & $7.0(0-17)$ & $\begin{array}{l}7.0 \\
(0-16)\end{array}$ \\
\hline Non-ventilated & $\mathrm{n} / \mathrm{a}$ & $\mathrm{n} / \mathrm{a}$ & $3.0(1-5)$ & $4.0(2-8)$ & & \\
\hline \multicolumn{7}{|l|}{ Original site of infection, $n(\%)$} \\
\hline cUTI & & & & & $3(12.0)$ & 0 \\
\hline cUTI with pyelonephritis & $7(36.8)$ & $4(44.4)$ & $\mathrm{n} / \mathrm{a}$ & $\mathrm{n} / \mathrm{a}$ & $\mathrm{n} / \mathrm{a}$ & $\mathrm{n} / \mathrm{a}$ \\
\hline cUTI without pyelonephritis & $4(21.1)$ & $1(11.1)$ & & & $\mathrm{n} / \mathrm{a}$ & $\mathrm{n} / \mathrm{a}$ \\
\hline $\begin{array}{l}\text { Acute uncomplicated } \\
\text { pyelonephritis }\end{array}$ & $8(42.1)$ & $4(44.4)$ & & & $\mathrm{n} / \mathrm{a}$ & $\mathrm{n} / \mathrm{a}$ \\
\hline Respiratory tract & $\mathrm{n} / \mathrm{a}$ & $\mathrm{n} / \mathrm{a}$ & & & & \\
\hline VAP & & & $4(50.0)$ & $7(70.0)$ & $2(8.0)$ & $2(15.4)$ \\
\hline HAP & & & $3(37.5)$ & $1(10.0)$ & 0 & 0 \\
\hline Ventilated HAP & & & 0 & $1(10.0)$ & 0 & 0 \\
\hline HCAP & & & $1(12.5)$ & $2(20.0)$ & 0 & 0 \\
\hline Ventilated HCAP & & & $1(12.5)$ & $2(20.0)$ & 0 & 0 \\
\hline $\mathrm{BSI} /$ sepsis & $\mathrm{n} / \mathrm{a}$ & $\mathrm{n} / \mathrm{a}$ & $\mathrm{n} / \mathrm{a}$ & $\mathrm{n} / \mathrm{a}$ & & \\
\hline cIAI & & & & & $4(16.0)$ & $2(15.4)$ \\
\hline SSSI & & & & & $2(8.0)$ & 0 \\
\hline IV line & & & & & $3(12.0)$ & $5(38.5)$ \\
\hline Other & & & & & $4(16.0)$ & $1(7.7)$ \\
\hline Unknown & & & & & $7(28.0)$ & $3(23.1)$ \\
\hline
\end{tabular}

APACHE II Acute Physiology and Chronic Health Evaluation II, BAT best available therapy, cIAI complicated intraabdominal infection, $C r C L$ creatinine clearance, $c U T I$ complicated urinary tract infection, $H A P$ hospital-acquired pneumonia, HCAP healthcare-associated pneumonia, $I V$ intravenous, $n / a$ not available, $S D$ standard deviation, $S O F A$ Sequential Organ Failure Assessment, SSSI skin and skin structure infection, VAP ventilator-associated pneumonia $N$, total number of patients with gram-negative bacteraemia at randomisation 
Table 3 Distribution of baseline gram-negative pathogens in blood at randomisation in the three studies (intention-to-treat population)

\begin{tabular}{|c|c|c|c|c|c|c|}
\hline & \multicolumn{2}{|c|}{ APEKS-cUTI ${ }^{\mathrm{a}}$} & \multicolumn{2}{|l|}{ APEKS-NP ${ }^{b}$} & \multicolumn{2}{|c|}{ CREDIBLE-CR $^{c}$} \\
\hline & $\begin{array}{l}\text { Cefiderocol } \\
(N=19) \\
N^{\prime}=19\end{array}$ & $\begin{array}{l}\text { Imipenem/cilastatin } \\
(N=9) \\
N^{\prime}=9\end{array}$ & $\begin{array}{l}\text { Cefiderocol } \\
(N=8) \\
N^{\prime}=9\end{array}$ & $\begin{array}{l}\text { Meropenem } \\
(N=10) \\
N^{\prime}=10\end{array}$ & $\begin{array}{l}\text { Cefiderocol } \\
(N=25) \\
N^{\prime}=27\end{array}$ & $\begin{array}{l}\text { BAT } \\
(N=13) \\
N^{\prime}=15\end{array}$ \\
\hline Enterobacterales, $n$ & 18 & 8 & 6 & 8 & 15 & 7 \\
\hline$K$. pneumoniae & 0 & 0 & 3 & 4 & 11 & 5 \\
\hline K. oxytoca & 1 & 0 & 0 & 0 & 1 & 0 \\
\hline E. coli & 16 & 8 & 1 & 1 & 3 & 0 \\
\hline S. marcescens & 0 & 0 & 2 & 1 & 0 & 0 \\
\hline E. aerogenes & 1 & 0 & 0 & 0 & 0 & 0 \\
\hline E. cloacae & 0 & 0 & 0 & 1 & 0 & 0 \\
\hline P. stuartii & 0 & 0 & 0 & 1 & 0 & 1 \\
\hline M. morganii & 0 & 0 & 0 & 0 & 0 & 1 \\
\hline Non-fermenters, $n$ & 1 & 1 & 3 & 2 & 12 & 8 \\
\hline Acinetobacter spp. & 0 & 1 & 2 & 0 & 12 & 6 \\
\hline$P$. aeruginosa & 1 & 0 & 0 & 1 & 0 & 2 \\
\hline B. cenocepacia & 0 & 0 & 1 & 1 & 0 & 0 \\
\hline
\end{tabular}

$B A T$ best available therapy

$N$, total number of patients with gram-negative bacteraemia at randomisation

$N$, total number of gram-negative pathogens from the blood

${ }^{a}$ APEKS-cUTI: $24 / 28$ (85.7\%) gram-negative pathogens were E. coli

bAPEKS-NP: $7 / 19$ (36.8\%) gram-negative pathogens were $K$. pneumoniae; $5 / 19$ (26.3\%) were non-fermenters

${ }^{\circ}$ CREDIBLE-CR: 17/42 (40.5\%) gram-negative pathogens were Klebsiella spp.; 18/42 (42.9\%) were Acinetobacter spp

\section{Bacteraemia and Clinical Outcomes}

Bacteraemia outcomes and clinical outcomes at EA are shown in Table 4. Across APEKS-cUTI, APEKS-NP and CREDIBLE-CR studies, on-therapy bacteraemia eradication rates were $100 \%$, $50.0 \%$ and $72.0 \%$ with cefiderocol and $77.8 \%$, $100 \%$ and $69.2 \%$ with comparator antibiotics, respectively. Persistence at EA was observed in a total of six patients (cefiderocol: $1 / 8$ patients [12.5\%] in APEKS-NP, 2/25 patients [8.0\%] in CREDIBLE-CR; comparators: $1 / 9$ patients [11.1\%] in APEKS-cUTI, 2/13 patients [15.4\%] in CREDIBLE-CR). Indeterminate bacteraemia response was observed in three patients $(37.5 \%)$ in APEKS-NP and five patients (20.0\%) in
CREDIBLE-CR with cefiderocol, and in one patient (11.1\%) in APEKS-cUTI and two patients (15.4\%) in CREDIBLE-CR with comparators, respectively. The clinical cure rates in APEKScUTI corresponded with bacteraemia eradication rates (cefiderocol: 100\% [19/19]; comparator: $77.8 \%[7 / 9])$. The clinical cure rate with cefiderocol treatment was 62.5\% [5/8] in APEKS$\mathrm{NP}$ and $64.0 \%$ [16/25] in CREDIBLE-CR (Table 4).

Bacteraemia and clinical outcomes at EOT and TOC are shown in Supplementary Table S1. At EOT, bacteraemia eradication rates were relatively high with all treatments across all three studies, except for cefiderocol $(37.5 \%$ [3/8]) in APEKS-NP and BAT $(46.2 \% \quad[6 / 13])$ in 
Table 4 Clinical and microbiological bacteraemia outcomes at early assessment by patient in the three studies (intention-totreat population)

\begin{tabular}{|c|c|c|c|c|c|c|}
\hline & \multicolumn{2}{|c|}{ APEKS-cUTI } & \multicolumn{2}{|l|}{ APEKS-NP } & \multicolumn{2}{|c|}{ CREDIBLE-CR } \\
\hline & $\begin{array}{l}\text { Cefiderocol } \\
(N=19)\end{array}$ & $\begin{array}{l}\text { Imipenem/ } \\
\text { cilastatin } \\
(N=9)\end{array}$ & $\begin{array}{l}\text { Cefiderocol } \\
(N=8)\end{array}$ & $\begin{array}{l}\text { Meropenem } \\
(N=10)\end{array}$ & $\begin{array}{l}\text { Cefiderocol } \\
(N=25)\end{array}$ & $\begin{array}{l}\text { BAT } \\
(N=13)\end{array}$ \\
\hline \multicolumn{7}{|c|}{ Bacteraemia outcome, $n(\%)$} \\
\hline Eradication & $19(100)$ & $7(77.8)$ & $4(50.0)$ & $10(100)$ & $18(72.0)$ & $9(69.2)$ \\
\hline Eradication & $14(73.7)$ & $6(66.7)$ & $3(37.5)$ & $6(60.0)$ & $16(64.0)$ & $9(69.2)$ \\
\hline Presumed eradication & $5(26.3)$ & $1(11.1)$ & $1(12.5)$ & $4(40.0)$ & $2(8.0)$ & 0 \\
\hline Persistence & 0 & $1(11.1)^{\mathrm{a}}$ & $1(12.5)^{\mathrm{b}}$ & 0 & $2(8.0)^{\mathrm{c}, \mathrm{d}}$ & $2(15.4)^{e, f}$ \\
\hline Indeterminate & 0 & $1(11.1)$ & $3(37.5)$ & 0 & $5(20.0)$ & $2(15.4)$ \\
\hline \multicolumn{7}{|l|}{ Clinical outcome, $n(\%)$} \\
\hline Cure & $19(100)$ & $7(77.8)$ & $5(62.5)$ & $9(90.0)$ & $16(64.0)$ & $4(30.8)$ \\
\hline Failure & 0 & $2(22.2)$ & $1(12.5)$ & $1(10.0)$ & $7(28.0)$ & $8(61.5)$ \\
\hline Indeterminate & 0 & 0 & $2(25.0)$ & $0(0)$ & $2(8.0)$ & $1(7.7)$ \\
\hline
\end{tabular}

$B A T$ best available therapy, $B S I$ bloodstream infection, $C R$ carbapenem resistant, $C S$ carbapenem susceptible, $c U T I$ complicated urinary tract infection, EOT end of treatment, $M I C$ minimum inhibitory concentration, TOC test of cure, $V A P$ ventilator-associated pneumonia

${ }^{a}$ One cUTI patient with $E$. coli (imipenem MIC $\leq 0.06 \mu \mathrm{g} / \mathrm{ml}$ ), blood isolate was eradicated at EOT and TOC

${ }^{\mathrm{b}}$ One VAP patient with CS $S$. marcescens (cefiderocol MIC $0.12 \mu \mathrm{g} / \mathrm{ml}$ ), blood isolate was eradicated at EOT and missing sample at TOC due to death

${ }^{\mathrm{c}}$ One BSI patient with CR $A$. baumannii (cefiderocol MIC $0.06 \mu \mathrm{g} / \mathrm{ml}$ ), no follow-up sample available because of death on Day 6 due to septic shock

${ }^{\mathrm{d} O n e}$ cUTI patient with cefiderocol-resistant (MIC $32 \mu \mathrm{g} / \mathrm{ml}$ ) and CR E. coli expressing NDM-5, following use of nephrostomy tube, blood isolate was eradicated at EOT and TOC

${ }^{\text {e}}$ One BSI patient (gentamicin treatment) with CS K. pneumoniae, blood isolate eradicated at EOT and TOC

${ }^{\mathrm{f}}$ One sepsis patient (colistin and ertapenem treatment) with CR A. baumannii and CS M. morganii, indeterminate bacteraemia response at EOT and TOC due to additional antibiotic

CREDIBLE-CR. Indeterminate responses with cefiderocol were seen in 10.5\% (2/19), 50.0\% (4/ $8)$ and $40.0 \%(10 / 25)$ patients in APEKS-cUTI, APEKS-NP and CREDIBLE-CR, respectively. Across all three studies, there was only one incidence of persistence (in a patient receiving imipenem-cilastatin; treatment was stopped on Day 2 due to an adverse event and early EOT assessment was performed) and one of recurrence, involving Burkholderia cenocepacia in a patient receiving cefiderocol in APEKS-NP. At TOC, there was no persistence across studies, and only one recurrence was recorded in
CREDIBLE-CR (i.e. recurrence of one CR E. coli isolate as a result of inadequate source control in a patient where hardware was left in place up to the TOC visit following surgery for pancreatic cancer, with development of intra-abdominal infection; blood samples at EA and EOT were negative). Indeterminate response rates for cefiderocol at TOC in APEKS-cUTI, APEKS-NP and CREDIBLE-CR were $21.1 \%, 87.5 \%$ and $52.0 \%$, respectively. In terms of clinical outcomes, in line with what was observed at EA, the highest cure rates with cefiderocol 
treatment were observed in APEKS-cUTI (EOT: 89.5\% [17/19]; TOC: 78.9\% [15/19]).

Bacteraemia and clinical outcomes by pathogen at EA, EOT and TOC for Enterobacterales and Acinetobacter spp. are shown in Supplementary Table S2. The highest eradication rates with cefiderocol were seen at EA and EOT in APEKS-cUTI for Enterobacterales, and these were maintained at the TOC visit. Clinical cure at TOC ranged from 16.7 to $100 \%$ for cefiderocol and from 42.9 to $62.5 \%$ for comparator agents. For Acinetobacter spp. infections in CREDIBLE-CR, eradication rates at EA, EOT and TOC were $75.0 \%(9 / 12), 58.3 \%(7 / 12)$ and $50.0 \%(6 / 12)$, respectively, with cefiderocol and
$66.7 \%(4 / 6), \quad 50.0 \%(3 / 6)$ and $50.0 \%(3 / 6)$, respectively, with BAT. Clinical cure rates at TOC were $33.3 \%(4 / 12)$ with cefiderocol and $50.0 \%(3 / 6)$ with BAT. Four $P$. aeruginosa isolates were identified: one in a patient receiving cefiderocol in APEKS-cUTI, one in a patient receiving meropenem in APEKS-NP and two in patients receiving BAT in CREDIBLE-CR. Three patients showed clinical cure and eradication at TOC, and one patient in the BAT arm showed clinical failure and had indeterminate bacteraemia outcome. Two B. cenocepacia isolates, both from APEKS-NP (one from each treatment arm), were clinical failures and had indeterminate microbiological responses at TOC.

Table 5 Outcomes by resistance mechanisms/extended-spectrum beta-lactamase for Enterobacterales in CREDIBLE-CR (intention-to-treat population)

\begin{tabular}{|c|c|c|c|}
\hline Resistance mechanism & Response, $n / N^{\prime}(\%)$ & Cefiderocol $(N=25)$ & BAT $(N=13)$ \\
\hline \multirow[t]{5}{*}{ Metallo-beta-lactamase (NDM, VIM, IMP) } & Eradication at EA & $3 / 4(75.0)$ & $1 / 2(50.0)$ \\
\hline & Persistence at EA & $1 / 4(25.0)$ & $0 / 2(0)$ \\
\hline & Clinical cure at TOC & $3 / 4(75.0)$ & $0 / 2(0)$ \\
\hline & Day 14 ACM & $0 / 4(0)$ & $0 / 2(0)$ \\
\hline & Day 28 ACM & $0 / 4(0)$ & $1 / 2(50.0)$ \\
\hline \multirow[t]{5}{*}{ Serine-beta-lactamase (KPC, OXA-48-like) } & Eradication at EA & $6 / 8(75.0)$ & $3 / 4(75.0)$ \\
\hline & Persistence at EA & $0 / 8(0)$ & $0 / 4(0)$ \\
\hline & Clinical cure at TOC & $4 / 8(50.0)$ & $2 / 4(50.0)$ \\
\hline & Day 14 ACM & $1 / 8(12.5)$ & $0 / 4(0)$ \\
\hline & Day 28 ACM & $1 / 8(12.5)$ & $2 / 4(50.0)$ \\
\hline \multirow[t]{5}{*}{$\mathrm{ESBL}^{\mathrm{a}}$} & Eradication at EA & $5 / 7(71.4)$ & $2 / 4(50.0)$ \\
\hline & Persistence at EA & $1 / 7(14.3)$ & $1 / 4(25.0)$ \\
\hline & Clinical cure at TOC & $4 / 7(57.1)$ & $1 / 4(25.0)$ \\
\hline & Day 14 ACM & $1 / 7(14.3)$ & $0 / 4(0)$ \\
\hline & Day 28 ACM & $1 / 7(14.3)$ & $2 / 4(50.0)$ \\
\hline
\end{tabular}

$A C M$ all-cause mortality, $B A T$ best available therapy, $E A$ early assessment, $E S B L$ extended-spectrum beta-lactamase, $I M P$ imipenemase metallo-beta-lactamase, KPC Klebsiella pneumoniae carbapenemase, NDM New Delhi metallo-beta-lactamase, $O X A$ oxacillinase, TOC test of cure, VIM Verona integron-encoded metallo-beta-lactamase

$N$, total number of patients with gram-negative bacteraemia at randomisation

$N$, total number of Enterobacterales isolates with the specific resistance mechanism(s)

a Sulphydryl variant of TEM extended-spectrum beta-lactamase (SHV), cefotaxime-hydrolysing beta-lactamase (CTX-M), cephamycin-hydrolysing beta-lactamase (CMY), Dhahran Hospital in Saudi Arabia $\beta$-lactamase (DHA) 
Bacteraemia and clinical outcomes by pathogen at EA, EOT and TOC for CR Enterobacterales and Acinetobacter spp. in CREDIBLECR are shown in Supplementary Table S3. At EA, eradication rates with cefiderocol and BAT were $75.0 \%(9 / 12)$ and $50.0 \%(3 / 6)$ for CR Enterobacterales and $70.0 \%(7 / 10)$ and $66.7 \%(4 / 6)$ for CR Acinetobacter spp., respectively. Clinical cure at TOC was seen in $50.0 \%(6 / 12)$ and $33.3 \%(2 / 6)$ patients with CR Enterobacterales infection and in $20.0 \%(2 / 10)$ and 50.0\% (3/6) patients with CR Acinetobacter spp. infection, respectively.

Details of the molecular information and baseline MICs for isolates with available data are shown in Supplementary Table S4. In APEKScUTI, the molecular information available for the six urine isolates (five ESBL-producing Enterobacterales and one OXA-23-producing CR A.baumannii) was anticipated to be applicable to the blood isolates based on equivalent susceptibility profiles. In APEKS-NP, among patients with secondary bacteraemia, one CR K. pneumoniae isolate was confirmed to be an ESBL and NDM-1 producer and one CR A. baumannii isolate expressing Guiana extendedspectrum beta-lactamase [GES] type. In CREDIBLE-CR, all isolates were CR due to expression of various ESBLs and carbapenemases.

Outcomes were summarised according to resistance mechanism/ESBL in Enterobacterales in CREDIBLE-CR and are shown in Table 5. Most isolates expressed a carbapenemase or an ESBL enzyme. Cefiderocol showed activity against MBLs (NDM, VIM, IMP); the rates of clinical cure at TOC and eradication at EA for Enterobacterales were both $75.0 \%(3 / 4)$.

Day-14 and Day-28 ACMs for patients with bacteraemia are shown in Supplementary Table S5. In the APEKS-cUTI study, there was one patient death $(5.3 \%$; a patient in the cefiderocol arm died as a result of a non-infection-related disease before end of treatment). Day 28 ACM rates with cefiderocol and comparators were $50.0 \%(4 / 8)$ and $30 \%(3 / 10)$, respectively, in APEKS-NP and 32.0\% (8/25) and $30.8 \%$ (4/13), respectively, in CREDIBLE-CR.

\section{DISCUSSION}

In this post hoc analysis of one pathogen-focused and two infection site-specific prospective randomised clinical trials, cefiderocol treatment resulted in a high degree of bacterial clearance from the bloodstream of patients with bacteraemia due to gram-negative pathogens, including CR isolates.

The studies were assessed individually because of considerable heterogeneity across the three studies, which precluded the pooling of data. As expected, patients in APEKS-cUTI had less severe illness than those in APEKS-NP and CREDIBLE-CR, both of which included critically ill patients receiving treatment in the intensive care unit. It was recognised when planning the analysis that this might lead to better outcomes in patients from APEKS-cUTI compared with those from the other two studies, particularly CREDIBLE-CR. However, because patients with known CR gram-negative pathogens at randomisation were excluded from APEKS-cUTI and APEKS-NP, most of the $\mathrm{CR}$ pathogens among patients with bacteraemia were identified in CREDIBLE-CR; therefore, results from patients with $\mathrm{CR}$ infections were not confounded by study design heterogeneity. Of note, the difference between the studies in the randomisation ratios $(2: 1$ for cefiderocol in APEKS-CUTI and CREDIBLE-CR, and 1:1 in APEKS-NP) explains the greater number of bacteraemia patients receiving cefiderocol $(n=52)$ than comparators $(n=32)$.

The bacterial species identified as the cause of bacteraemia varied greatly by study population, with $86 \%$ of patients in APEKS-cUTI having E. coli, whereas K. pneumoniae was most frequent in APEKS-NP and A. baumannii in CREDIBLE-CR. The infection source is important when considering comparison of outcomes between different clinical studies.

The eradication rate of gram-negative pathogens was highest in APEKS-cUTI, although early bacteraemia eradication rates (i.e. those within 3-4 days of cefiderocol initiation) were very high in each study. Eradication of the baseline pathogen was independent of carbapenem susceptibility status. Enterobacterales 
isolates with MBLs were eradicated with cefiderocol at a high rate $(75 \%$ [3/4]) at EA and 100\% $(4 / 4)$ at EOT. This is consistent with in vitro data showing activity of cefiderocol in the presence of beta-lactamases, including MBLs, as well as porin mutations and upregulated efflux pumps [29]. Across studies, rates of confirmed persistence were low, even at EA. It is likely that the one instance of recurrence at TOC was due to inadequate source control in a cancer patient rather than due to antibiotic failure. By TOC, in CREDIBLE-CR there was no persistence.

In terms of individual pathogens, the ontherapy eradication of Acinetobacter spp. from blood, found primarily in CREDIBLE-CR, suggests that cefiderocol may be an effective antibiotic against this difficult-to-treat pathogen in patients with bacteraemia. As reported previously [28], in the CREDIBLE-CR study, Acinetobacter spp. infections were associated with a higher mortality rate than infections due to other pathogens, which was explained in part by prior or ongoing shock at randomisation and a high rate of intensive care unit admission [28]. In the current analysis, the Day $28 \mathrm{ACM}$ rates in CREDIBLE-CR were $32.0 \%$ with cefiderocol and $30.8 \%$ with BAT for patients with bacteraemia, a subgroup that included a few patients with the primary diagnosis of NP or CUTI, and excluded patients with the diagnosis of sepsis without positive blood culture, potentially influencing mortality rates.

Given the source and severity of the infections and the condition of patients involved in the different studies, it is not surprising that the mortality rates among patients with bacteraemia in APEKS-NP and CREDIBLE-CR were higher than those in APEKS-cUTI. The higher mortality rates may be a reflection of risk factors such as pneumonia, ventilation status, high APACHE II and SOFA scores in APEKS-NP and CR gram-negative infections in CREDIBLE-CR. Mortality rates are known to be affected by primary infection severity $[10,30]$ and infection site. In gram-negative BSI, including CR Enterobacterales, mortality was found to be higher in patients with a pulmonary source of infection compared with other sources, including UTI $[1,2,7,14]$. These factors may help to explain the difference between our findings and those from the MERINO trial, in which the overall mortality rate remained $<10 \%$, despite a mortality difference between the meropenem and piperacillin-tazobactam arms [7]. The MERINO trial excluded severely ill patients, the main source of bacteraemia was the urinary tract (64\%) and E. coli was the causative pathogen in around $86 \%$ of patients [7].

Strengths of the current analysis include the use of data from three prospective randomised clinical trials, with consistent and standardised microbiological sample collection, use of central laboratory and data analysis and inclusion of patients with a variety of MDR and CR gramnegative pathogens and a variety of infections that required hospitalisation and intravenous antibiotic treatment. There was a broad range of bacterial species, both Enterobacterales and non-fermenters (most importantly Acinetobacter), as well as considerable diversity in bacterial resistance mechanisms, as assessed by molecular analysis. Of note, cefiderocol treatment was given as monotherapy to 47 of 52 (90.4\%) patients across the studies, providing a clearer link between cefiderocol therapy and outcomes.

One of the main limitations of our analysis is the inconsistent collection of post-randomisation blood cultures for patients with a positive blood culture at randomisation, particularly in APEKS-cUTI and APEKS-NP. This means that a true rate of microbiological eradication or, conversely, persistence could be underestimated when responses are classified as indeterminate. Among the studies reported here, the highest rate of repeat culture provision was in the BSI/sepsis subset of patients in CREDIBLECR. We found a high indeterminate microbiological response rate in APEKS-NP due to deaths, additional antibiotics and missing samples. While the lack of repeat cultures in our analysis was unavoidable given the design of the studies analysed, use of an algorithm that allowed clinical response to determine bacteraemia response helped in part to address the problem of indeterminate responses. As stated previously, pooled analysis of data was not feasible because of the heterogeneity across the studies. Finally, any interpretation of the data should acknowledge its descriptive nature and the small size of the subgroups. 
The analysis highlights the need for better protocol compliance with on- and post-therapy blood cultures [31-33] in randomised clinical trials involving site-specific infections with concomitant bacteraemia to provide a more accurate assessment of bacteraemia clearance. Our post hoc analysis data are complemented by efficacy findings for cefiderocol in real-life cases of difficult-to-treat severe gram-negative infections. Microbiological clearance from repeat blood cultures was seen in around $67 \%$ of a total of 60 patients with BSI due mainly to CR or XDR A. baumannii and difficult-to-treat resistant $P$. aeruginosa [34-42]. A more complete picture of the real-world efficacy of cefiderocol in gram-negative BSI patients will be provided by the ongoing Phase 2 GAMECHANGER study, an open-label, prospective, randomised, controlled, non-inferiority trial, comparing cefiderocol and standard of care in the treatment of healthcare-associated or hospital-acquired BSI (NCT03869437; [43]).

\section{CONCLUSION}

The results of this post hoc analysis suggest that cefiderocol may be an option for the treatment of gram-negative bacteraemia; cefiderocol eliminated gram-negative bacteraemia within 3-4 days in a large proportion of patients and rates of confirmed persistence and recurrence at EOT or TOC were very low. Based on these results, further prospective clinical trials to define the treatment benefit of cefiderocol for gram-negative bacteraemia are indicated.

\section{ACKNOWLEDGEMENTS}

Funding. Sponsorship for this analysis and Rapid Service Fee were funded by Shionogi.

Medical Writing Assistance. Editorial assistance in the preparation of this article was provided by Dr. Adrienn Kis and Dr. Joanne Shrewsbury-Gee of Highfield, Oxford, UK. Support for this assistance was funded by Shionogi.
Authorship. All named authors meet the International Committee of Medical Journal Editors (ICMJE) criteria for authorship for this article, take responsibility for the integrity of the work as a whole, and have given their approval for this version to be published.

Author Contributions. Masahiro Kinoshita, Takamichi Baba, Simon Portsmouth, and Roger Echols participated in the conceptualisation, data collection, data analysis, data interpretation, drafting and editing of the manuscript. David Paterson participated in the data interpretation, drafting and editing of the manuscript. All authors approved the final version for submission.

Prior Presentation. Data in this manuscript were presented in part at IDWeek 2020. Paterson D, Kinoshita M, Toyoizumi K, Matsunaga Y, Echols R. Outcomes in Patients with GramNegative Bacteremia from Phase 2 and Phase 3 Clinical Trials of Cefiderocol, a Novel Siderophore Cephalosporin. IDWeek 2020, 21-25 October 2020; www.idweek.org; Poster 1285.

Disclosures. Masahiro Kinoshita and Takamichi Baba declare that they are employees of Shionogi \& Co., Ltd., Osaka, Japan. Simon Portsmouth declares that he is employee of Shionogi Inc., Florham Park, NJ, USA. David Paterson has received consultancy fee from Shionogi \& Co., Ltd., Osaka, Japan. Roger Echols has received consultancy fee from Shionogi \& Co., Ltd., Osaka, Japan.

Compliance with Ethics Guidelines. Data from all three studies included in this post hoc analysis have been reported elsewhere. For each trial, the study protocol was approved by the institutional review board or ethics committee at each participating centre and complied with International Conference on Harmonisation Guideline for Good Clinical Practice and the Helsinki Declaration of 1964 and its later amendments. All patients or their representatives provided written informed consent according to local guidelines. 
Data Availability. All data generated or analysed during this study are included in this published article or supplementary information files.

Open Access. This article is licensed under a Creative Commons Attribution-NonCommercial 4.0 International License, which permits any non-commercial use, sharing, adaptation, distribution and reproduction in any medium or format, as long as you give appropriate credit to the original author(s) and the source, provide a link to the Creative Commons licence, and indicate if changes were made. The images or other third party material in this article are included in the article's Creative Commons licence, unless indicated otherwise in a credit line to the material. If material is not included in the article's Creative Commons licence and your intended use is not permitted by statutory regulation or exceeds the permitted use, you will need to obtain permission directly from the copyright holder. To view a copy of this licence, visit http:// creativecommons.org/licenses/by-nc/4.0/.

\section{REFERENCES}

1. Thaden JT, Park LP, Maskarinec SA, Ruffin F, Fowler VG Jr, van Duin D. Results from a 13-year prospective cohort study show increased mortality associated with bloodstream infections caused by Pseudomonas aeruginosa compared to other bacteria. Antimicrob Agents Chemother. 2017;61(6):e02671e2716.

2. Hovan MR, Cederbaum VK, Kirn TJ. Predictors of mortality in carbapenem-resistant Enterobacterales bacteremia. In: Presented at IDWeek 2020. 2020. www.idweek.org; Poster \#304.

3. van Staaden H, Hendricks C, Spicer K. Bacteraemia and antibiotic sensitivity in a tertiary neonatal intensive care unit. S Afr J Infect Dis. 2021;36(1): 195.

4. Folgori L, Di Carlo D, Comandatore F, et al. Antibiotic susceptibility, virulome, and clinical outcomes in European infants with bloodstream infections caused by Enterobacterales. Antibiotics (Basel). 2021;10(6):706.
5. Chan JD, Bryson-Cahn C, Kassamali-Escobar Z, Lynch JB, Schleyer AM. The changing landscape of uncomplicated Gram-negative bacteremia: a narrative review to guide inpatient management. J Hosp Med. 2020;15:746-53.

6. Yahav D, Franceschini E, Koppel F, et al. Seven versus 14 days of antibiotic therapy for uncomplicated Gram-negative bacteremia: a noninferiority randomized controlled trial. Clin Infect Dis. 2019;69:1091-8.

7. Harris PNA, Tambyah PA, Lye DC, et al. Effect of piperacillin-tazobactam vs meropenem on 30-day mortality for patients with E. coli or Klebsiella pneumoniae bloodstream infection and ceftriaxone resistance: a randomized clinical trial. JAMA. 2018;320(10):984-94. (Erratum in: JAMA. 2019;321(23):2370).

8. Shields RK, Zhou Y, Kanakamedala H, Cai B. Burden of illness in US hospitals due to carbapenem-resistant Gram-negative urinary tract infections in patients with or without bacteraemia. BMC Infect Dis. 2021;21(1):572.

9. Timsit J-F, Ruppé E, Barbier F, Tabah A, Bassetti M. Bloodstream infections in critically ill patients: an expert statement. Intensive Care Med. 2020;46: 266-84.

10. Blot S. Setting the baseline to fight Gram-negative bacteraemia: the necessity of epidemiological insights. Infect Dis (Lond). 2019;51(1):23-5.

11. Porat Y, Nashashibi J, Poran I, Paul M. Predictors of readmission following discharge of patients with Gram-negative bacteremia: a retrospective cohort study. Open Forum Infect Dis. 2021;8(8):ofab373.

12. Son HJ, Cho EB, Bae M, et al. Clinical and microbiological analysis of risk factors for mortality in patients with carbapenem-resistant Acinetobacter baumannii bacteremia. Open Forum Infect Dis. 2020;7(10):ofaa378.

13. Sader HS, Castanheira M, Huband MD, Shortridge D, Carvalhaes CG, Mendes RM. Bloodstream infections in the United States and Europe: etiology and antimicrobial susceptibility results from the SENTRY Antimicrobial Surveillance Program (2016-2019). Presented at IDWeek 2020. 2020. www.idweek.org; Oral presentation \#37.

14. Santoro A, Franceschini E, Meschiari M, et al. Epidemiology and risk factors associated with mortality in consecutive patients with bacterial bloodstream infection: impact of MDR and XDR bacteria. Open Forum Infect Dis. 2020;7(11): ofaa 461. 
15. Cai B, Tillotson G, Benjumea D, Callahan P, Echols R. The burden of bloodstream infections due to Stenotrophomonas maltophilia in the United States: a large, retrospective database study. Open Forum Infect Dis. 2020;7(5):ofaa141.

16. Papadimitriou-Olivgeris M, Fligou F, Spiliopoulou A, et al. Risk factors and predictors of carbapenemresistant Pseudomonas aeruginosa and Acinetobacter baumannii mortality in critically ill bacteraemic patients over a 6-year period (2010-15): antibiotics do matter. J Med Microbiol. 2017;66:1092-101.

17. Kadri SS, Adjemian J, Lai YL, et al. Difficult-to-treat resistance in Gram-negative bacteremia at 173 US hospitals: retrospective cohort analysis of prevalence, predictors, and outcome of resistance to all first-line agents. Clin Infect Dis. 2018;67:1803-14.

18. Falcone M, Daikos GL, Tiseo G, et al. Efficacy of ceftazidime-avibactam plus aztreonam in patients with bloodstream infections caused by metallo- $\beta$ lactamase-producing Enterobacterales. Clin Infect Dis. $2021 ; 72(11): 1871-8$.

19. Bassetti M, Vena A, Sepulcri C, Giacobbe DR, Peghin M. Treatment of bloodstream infections due to Gram-negative bacteria with difficult-to-treat resistance. Antibiotics. 2020;9:632.

20. Sands K, Carvalho MJ, Portal E, et al. Characterization of antimicrobial-resistant Gram-negative bacteria that cause neonatal sepsis in seven low- and middle-income countries. Nat Microbiol. 2021;6(4): 512-23.

21. Laxminarayan R, Bhutta ZA. Antimicrobial resistance-a threat to neonate survival. Lancet Glob Health. 2016;4:e676-7.

22. Maskarinec SA, Park LP, Ruffin F, et al. Positive follow-up blood cultures identify high mortality risk among patients with Gram-negative bacteraemia. Clin Microbiol Infect. 2020;26:904-10.

23. Ito A, Kohira $\mathrm{N}$, Bouchillon $\mathrm{SK}$, et al. In vitro antimicrobial activity of S-649266, a catechol-substituted siderophore cephalosporin, when tested against non-fermenting Gram-negative bacteria. J Antimicrob Chemother. 2016;71:670-7.

24. Delgado-Valverde M, Conejo MDC, Serrano L, Fernández-Cuenca F, Pascual A. Activity of cefiderocol against high-risk clones of multidrug-resistant Enterobacterales, Acinetobacter baumannii, Pseudomonas aeruginosa and Stenotrophomonas maltophilia. J Antimicrob Chemother. 2020;75:1840-9.

25. Hackel MA, Tsuji M, Yamano Y, Echols R, Karlowsky JA, Sahm DF. In vitro activity of the siderophore cephalosporin, cefiderocol, against a recent collection of clinically relevant Gram-negative bacilli from North America and Europe, including carbapenem-nonsusceptible isolates (SIDERO-WT2014 Study). Antimicrob Agents Chemother. 2017;61(9):e00093-e117.

26. Portsmouth S, van Veenhuyzen D, Echols R, et al. Cefiderocol versus imipenem-cilastatin for the treatment of complicated urinary tract infections caused by Gram-negative uropathogens: a phase 2, randomised, double-blind, non-inferiority trial. Lancet Infect Dis. 2018;18:1319-28.

27. Wunderink RG, Matsunaga Y, Ariyasu M, et al. Cefiderocol versus high-dose, extended-infusion meropenem for the treatment of Gram-negative nosocomial pneumonia (APEKS-NP): a randomised, double-blind, phase 3, non-inferiority trial. Lancet Infect Dis. 2021;21:213-25.

28. Bassetti M, Echols R, Matsunaga Y, et al. Efficacy and safety of cefiderocol or best available therapy for the treatment of serious infections caused by carbapenem-resistant Gram-negative bacteria (CREDIBLE-CR): a randomised, open-label, multicentre, pathogen-focused, descriptive, phase 3 trial. Lancet Infect Dis. 2021;21:226-40.

29. Ito $\mathrm{A}$, Sato $\mathrm{T}$, Ota $\mathrm{M}$, et al. In vitro antibacterial properties of cefiderocol, a novel siderophore cephalosporin, against Gram-negative bacteria. Antimicrob Agents Chemother. 2017;62: e01454-e1517.

30. Dillon R, Khankhel Z, De Anda C, Bruno C, Puzniak L. Ceftolozane/tazobactam (Zerbaxa) for the treatment of Pseudomonas aeruginosa (PSA) bacteraemia: a systematic literature review (SLR). In: Presented at IDWeek 2020. 2020. www.idweek.org; Poster \#1596.

31. Cogliati Dezza F, Curtolo A, Volpicelli L, Ceccarelli G, Oliva A, Venditti M. Are follow-up blood cultures useful in the antimicrobial management of Gram negative bacteremia? A reappraisal of their role based on current knowledge. Antibiotics (Basel). 2020;9:895.

32. Doern GV, Carroll KC, Diekema DJ, et al. Practical guidance for clinical microbiology laboratories: a comprehensive update on the problem of blood culture contamination and a discussion of methods for addressing the problem. Clin Microbiol Rev. 2019;33(1):e00009-19.

33. Spaziante M, Oliva A, Ceccarelli G, Alessandri F, Pugliese F, Venditti M. Follow-up blood cultures in Gram-negative bacilli bacteremia: are they needed for critically ill patients? Minerva Anestesiol. 2020;86:498-506.

34. Bavaro DF, Belati A, Diella L, et al. Cefiderocolbased combination therapy for "difficult-to-treat" 
Gram-negative severe infections: real-life case series and future perspectives. Antibiotics (Basel). 2021;10:65.

35. Gras J, Villar-Fernandez S, Baylac P, et al. Successful cefiderocol therapy of severe infections due to difficult-to-treat Pseudomonas aeruginosa in two allogeneic hematopoietic stem cell transplantation recipients. Ann Hematol. 2022. https://doi.org/10. 1007/s00277-021-04737-z.

36. Meschiari M, Volpi S, Faltoni M, et al. Real-life experience with compassionate use of cefiderocol for difficult-to-treat resistant Pseudomonas aeruginosa (DTR-P) infections. JAC Antimicrob Resist. 2021;3(4):dlab188. https://doi.org/10.1093/jacamr/ dlab188.

37. Pascale R, Pasquini Z, Bartoletti M, et al. Cefiderocol treatment for carbapenem-resistant Acinetobacter baumannii infection in the ICU during the COVID19 pandemic: a multicentre cohort study. JAC Antimicrob Resist. 2021;3(4):dlab174. https://doi. org/10.1093/jacamr/dlab174.

38. Gatti M, Bartoletti M, Cojutti PG, et al. A descriptive case series of pharmacokinetic/pharmacodynamic target attainment and microbiological outcome in critically ill patients with documented severe extensively drug-resistant Acinetobacter baumannii bloodstream infection and/or ventilator-associated pneumonia treated with cefiderocol. J Glob Antimicrob Resist. 2021;27:294-8.

39. Falcone M, Tiseo G, Nicastro M, et al. Cefiderocol as rescue therapy for Acinetobacter baumannii and other carbapenem-resistant Gram-negative infections in intensive care unit patients. Clin Infect Dis. 2021;72:2021-4.

40. Oliva A, Ceccarelli G, De Angelis M, et al. Cefiderocol for compassionate use in the treatment of complicated infections caused by extensively and pan-resistant Acinetobacter baumannii. J Glob Antimicrob Resist. 2020;23:292-6.

41. Edgeworth JD, Merante D, Patel S, et al. Compassionate use of cefiderocol as adjunctive treatment of native aortic valve endocarditis due to extremely drug-resistant Pseudomonas aeruginosa. Clin Infect Dis. 2019;68:1932-4.

42. Trecarichi EM, Quirino A, Scaglione V, et al. Successful treatment with cefiderocol for compassionate use in a critically ill patient with XDR Acinetobacter baumannii and KPC-producing Klebsiella pneumoniae: a case report. J Antimicrob Chemother. 2019;74:3399-401.

43. Wright $\mathrm{H}$, Harris PNA, Chatfield MD, et al. Investigator-driven randomised controlled trial of cefiderocol versus standard therapy for healthcareassociated and hospital-acquired Gram-negative bloodstream infection: study protocol (the GAME CHANGER trial): study protocol for an open-label, randomised controlled trial. Trials. 2021;22:889.

\section{Publisher's Note}

Springer Nature remains neutral with regard to jurisdictional claims in published maps and institutional affiliations. 